\title{
Studies of Different Swarm Modes for the MNPs under the Rotating Magnetic Field
}

\author{
Kiana Abolfathi, Mohammad Reza Hairi Yazdi, and Ali Kafash Hoshiar*, IEEE member
}

\begin{abstract}
The principal constraint in the application of the microrobots in drug delivery is swarm control. The rotating magnetic field has shown the potential of capturing and controlling of the swarm of magnetic nanoparticlebased microrobots. Despite various experimental studies to capture the swarm of the magnetic nanoparticle-based microrobot in a rotating magnetic field, a simulation platform for the swarm of aggregated magnetic nanoparticles (MNPs) has not been introduced. This study proposes a simulation platform to study the swarm of aggregated magnetic nanoparticles in a rotating field. An experimental setup was developed to investigate the different swarm modes in a rotating magnetic field, and the results show an agreement between the experimental and simulation results for the micro- and nanoparticles. The effects of the environmental parameters (initial dispersion of nanoparticles), process parameters (magnetic field intensity, frequency, actuation time), and geometrical parameters (particle diameter) were studied to describe the swarm behavior in a rotating magnetic field. These studies revealed the role of each parameter in creating the swarm and showed how the size of aggregates can be controlled. The presented approach can be used to design the magnetic nanoparticle-based microrobots effectively.
\end{abstract}

Index Terms-Magnetic nanoparticles, Microrobots, Swarm steering, Rotating magnetic field, Targeted Drug Delivery.

\section{INTRODUCTION}

$\mathrm{T}^{\mathrm{N}}$ recent decades, magnetic nanoparticles (MNPs) have learned widespread interest in biomedical applications, and significant progress is made in the development of MNPs for medical applications [1], [2]. MNPs based targeted drug delivery (TDD) is used to elevate the concentration of the drug in a location of interest inside the body. In this method, the drug-coated MNPs are injected into the body, and an external magnetic field is used to elevate their concentration in a region of interest [1]. The applications of this method vary from drug delivery to tumors [2], and infection area [3], to opening the blood clots [4]. Recent development in magnetic particles for

K. Abolfathi and M.R. Hairi Yazdi are with the School of Mechanical Engineering, University of Tehran, P.C. 1439955961, Tehran, Iran (email: kiana.abolfathi@ut.ac.ir, myazdi@ut.ac.ir).

* (Corresponding Author) A. K. Hoshiar is with the School of Computer Science and Electronic Engineering, University of Essex, Colchester, P.C. CO4 3SQ, UK (email: A.kafashhoshiar@essex.ac.uk). both imaging and therapeutic effects [5] as well as new approaches for in vivo targeted drug delivery [6], made the study of the swarm of nanoparticles necessary. To have efficient performance, the MNPs should be steered in the swarm shape to increase the concentration of the MNPs in the region of interest.

The Swarm of MNPs can deliver the required dose of drugs to the targeted region [7]. However, there are two major limiting factors. First, all of the particles inside a magnetic field will experience the same force and torque, which makes the individual nanoparticles control difficult. Second, no arrangement of the external static magnetic field could concentrate the particles at a location of interests [8], [9].

The magnetic actuation schemes have been developed to steer the micro devices and magnetic nanoparticles. Various actuation schemes based on the magnetic resonance imaging (MRI) systems and electromagnets actuation (EMA) systems were developed to evaluate the performance and accuracy of the steering [10], [11]. The gradient steering based on MRI was developed [12]. In these studies, by controlling the gradient field, the magnetic objects, were controlled [7], [12]-[14]. Although due to the first limitation simultaneous control of multiple devices is hard to achieve, swarm steering with the functionalization of the magnetic field was achieved.

Dynamic magnetic actuation by electromagnetic systems is also developed to concentrate MNPs in a location of interest [15], [16]. The electromagnetic actuation and permanent magnetic actuation are used to elevate the concentration of nanoparticles in a region of interest [17], [18]. The magnetic scheme to steer swarm of nanoparticles in the multi bifurcations were developed [19], [20]. Although swarm steering was achieved, the inefficient magnetic gradient-based control in these systems was a limiting factor.

To overcome this limitation, the rotating magnetic field for concentrating nanoparticles [21], and dynamic actuation for separating them is used. Moreover, the rotating magnetic field for steering the vortex-like swarm has been introduced [22]. By using a predefined magnetic field, a method of controllable disassembly of a paramagnetic nanoparticle chain has been reported [23]. The rotating magnetic field has been introduced to generate vortex-like MNPs from the dispersed nanoparticles [24], [25]. A strategy to reconfigure paramagnetic nanoparticles into ribbon-like swarms by the oscillating magnetic field has been reported [26]. The simulation platform developed for the aggregated MNPs steering was limited to the gradient fieldbased steering [27], [28]. In this study, we developed a computational platform to show the swarm behavior under a rotating magnetic field. 


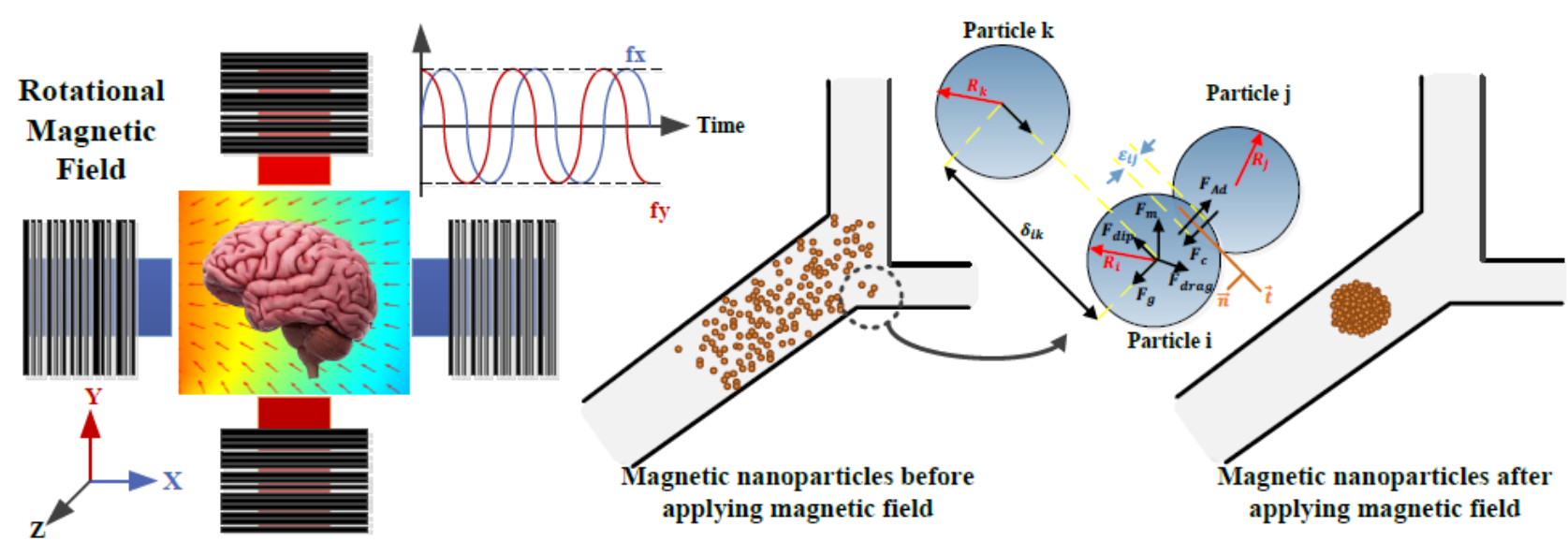

Figure 1.Conceptual schematic for the aggregated magnetic nanoparticles under the dynamic magnetic fields, and the free body diagram of the magnetic nanoparticles

Fig. 1 shows the conceptual design for a rotating magnetic field to generate the aggregate formation for the swarm steering. In this study, a computational platform was developed to study the aggregation formation. By using the governing dynamics of the particles, the simulation platform shows different swarm modes. Experimental results are used to compare the simulation data. The efficient parameters to generate different swarm modes have been studied.

The remaining of the paper is divided into three sections. First, the governing dynamics is developed. Next, a simulation platform based on the governing dynamics was developed, and different swarm modes have been studied by using the experimental setup. Finally, the effects of the environmental parameters, process parameters, and geometrical parameters have been studied.

\section{EQUATIONS OF MOTION}

In this section, the effective forces in the swarm of the magnetic nanoparticles have been introduced. All nanoparticles are assumed to have the same spherical shape and dimensions. In the modeling, the particles are studied separately, and we analyzed the forces applied to each particle. The schematic of the forces applied to a particle is depicted in Fig. 1.

The particle size plays a principal role in the Brownian force. When the particle size is less than $0.1 \mu \mathrm{m}$, the Brownian force will affect the motion of the particles [29]. Although using small particles $(<10 \mathrm{~nm})$ has significant benefit in clinical applications, such as drug delivery and hyperthermia, the steering of the small particles experimentally is hard to achieve due to the force limitation. In this study, the particle size is limited to $>100 \mathrm{~nm}$. Consequently, the Brownian motion is neglected in the modeling of the system. The Newtonian dynamics is used to derive the equations of motions for the movement of the nanoparticles:

$$
m_{i} \dot{v}_{i}=F_{m_{i}}+F_{\text {dip }_{i}}+F_{\text {drag }_{i}}+F_{C A d_{i}}+F_{G_{i}}
$$

where the index $i$ represents the $i^{\text {th }}$ particle. $v_{i}$ denotes the particle velocity, and $\dot{v}_{i}$ denotes the particle acceleration, $m_{i}$ represents the mass of the particle. The result of the magnetic forces on the particle is shown by $F_{m_{i}} . F_{d i p_{i}}$ represents the sum of the dipole forces between the $\mathrm{i}^{\text {th }}$-particle and other nanoparticles. $F_{\text {drag }_{i}}$ represents the drag forces exerted on the particle. $F_{C A d_{i}}$ represents the contact forces and adhesion forces between the particles. $F_{G_{i}}$ represents the set of gravitational forces exerted on the particles, and is equal to $\pi d_{i}^{3}\left(\rho_{i}-\rho_{f}\right) / 6$, $\rho_{i}$ and $\rho_{f}$ are the particle and fluid density. Due to the small diameter of the particles, the gravity force is negligible. In the following, we will elaborate on the influential force.

\section{A. Magnetic Force}

An external magnetic field has been used to capture the nanoparticles. The magnetic force is the result of the magnetic field gradient and introduced as [15]:

$$
F_{m_{i}}=V \mu_{r} M_{\text {sat }} \cdot \nabla H_{i}
$$

where, $V$ is the volume the nanoparticles, $\mu_{r}$ represents the permeability coefficient of the environment, $M$ represents the magnetic polarization (If the magnetic field is strong, the magnitude of the magnetic polarity will be equal to the finite limit indicated by the symbol $M_{\text {sat }}$ ) and $H$ is magnetic field intensity. $\nabla$ is the gradient operator. The Eq. (2) shows that the magnetic force is proportional to the volume of the nanoparticles. Therefore, the decrease in the particle radius will lead to a decrease in force and makes the smaller particles less responsive to the magnetic field.

The placement of the magnetic particles under a magnetic field results in the polarity of the particles. The phenomenon of polarity in the particles creates a magnetic force among the particles. The dipole force plays a key role in the formation of the aggregations. The dipole force is modeled as [30]:

$$
F_{d i p_{i}}=\sum_{j}^{N} F_{d i p_{i j}}
$$

where the index $\mathrm{i}$ and $\mathrm{j}$ denote the $\mathrm{i}^{\text {th }}$ and $\mathrm{j}^{\text {th }}$ particles, and $N$ denotes the number of the nanoparticles. $F_{d i p_{i j}}$ denotes the dipole force of $\mathrm{j}^{\text {th }}$ particle on the $\mathrm{i}^{\text {th }}$ particle. The dipole force between two particles $i$ and $j$ have equal magnitudes and opposite directions $\left(F_{d i p_{i j}}=-F_{d i p_{j i}}\right)$. Dipole force is modeled as follows [30]: 


$$
\begin{gathered}
F_{d i p_{i k}}=\frac{3 \mu_{0}\left|\widehat{m}_{i} \| \widehat{\mid}_{k}\right|}{4 \pi \hat{r}_{k i}^{4}}\left(\left(\hat{r}_{i k}\left(\widehat{m}_{i} \cdot \widehat{m}_{k}\right)+\widehat{m}_{i}\left(\hat{r}_{i k} \cdot \widehat{m}_{k}\right)+\right.\right. \\
\left.\hat{m}_{k}\left(\hat{r}_{i k} \cdot \hat{m}_{i}\right)-5 \hat{r}_{k i}\left(\hat{r}_{k i} \cdot \widehat{m}_{i}\right)\left(\hat{r}_{k i} \cdot \widehat{m}_{k}\right)\right)
\end{gathered}
$$

where, $\mu_{0}$ represents the permeability coefficient of the environment. $r_{i k}$ represents the distance vector between center of the particle $i$ to the center of the particle $k$ and $r_{k i}$ represents the distance vector between center of the particle $k$ to the center of the particle $i . m_{i}$ and $m_{k}$ are the magnetic moments of the particle $i$ and $k$, respectively. The magnetic moment for the particle $i$ is $m_{i}=\frac{4 \pi R_{i}^{3}}{\mu_{0}}\left(\frac{\mu_{r}-1}{\mu_{r}+1}\right) B_{i}$ and $B_{i}$ is the field of the particle $i$ that is calculated by the following formula:

$$
B_{i}=B_{e x t}+\sum_{k=1, k \neq i}^{N} B_{d i p_{k i}}
$$

$\mathrm{B}$ is the magnitude of the external field, $B_{d i p_{k i}}$ is the magnetic intensity due to the polarity of the $\mathrm{k}^{\text {th }}$ particle to the $\mathrm{i}^{\text {th }}$ particle which is obtained by the following formula:

$$
B_{k i}=\left(\frac{\mu_{r}-1}{\mu_{r}+1}\right) R_{i}^{3} \sum_{k=1, k \neq i}^{N} \frac{3\left(B_{i} \cdot \hat{r}_{k i}\right)}{\hat{r}_{k i}^{5}}-\frac{B_{k}}{\hat{r}_{k i}^{3}}
$$

In general, by expanding the equation above and writing the field in the $x, y$ and $z$ directions, we derive a $3 N$ equations and $3 N$ parameters system. Solving this equation gives the values of $B_{i}$. By placing $B_{i}$ in the equation, the value of the $\mathrm{i}^{\text {th }}$ particle will be obtained.

The effect of the dipole force decreases with the distance [27]. Therefore, to simplify the simulation, the dipole force for particles with a distance greater than $8 r$ ( $r$ is particle radius) is considered to be zero. The length and width of the initial particles scattering space are assumed as a constant:

$$
L=6 r_{\text {ave }} \operatorname{round}(\sqrt{N})
$$

$\mathrm{N}$ is the number of particles and $r_{\text {ave }}$ is the average particle radius.

\section{B. Contact Force and Adhesion Force}

The $F_{C A d_{i}}$ is sum of the contact and adhesion force applied to the $\mathrm{i}^{\text {th }}$ particle $\left(F_{C A d_{i}}=F_{C_{i}}+F_{A d_{i}}\right)$. In the Fig 1 the parameter $\delta_{i j}$ represents the distance between the centers of the two particles $i$ and $j$. If $\delta_{i j}<R_{i}+R_{j}$, then we will have contact force. The Hertzian contact model is used to model the contact force, which is defined as follows:

$$
F_{C_{i}}=-F_{C_{j}}=K \varepsilon_{i j}^{1.5}
$$

where $\mathrm{K}$ is the spring constant and $\varepsilon_{i j}$ represents the interaction between the particle $i$ and $j$ in contact with each other and its value is $\delta_{i j}-\left(R_{i}+R_{j}\right)$.

The adhesion force is modeled by the following formula:

$$
F_{A d_{i}}=\tau \pi\left(\frac{3 F_{c_{i}} d_{i j}^{*}}{8 E_{i j}^{*}}\right)^{\frac{2}{3}}
$$

where, the $i$ and $j$ represent $\mathrm{i}^{\text {th }}$ and $\mathrm{j}^{\text {th }}$ particles that are in contact with each other. $\tau$ is equal to the adhesion energy. $F_{c_{i}}$ represents the contact force applied to the particle $i$ due to the contact with particle $j$. According to Eq. (9) for the particle adhesion force, we have $F_{A d_{i}}=-F_{A d_{j}}$, where $E_{i j}^{*}$ is the equivalent elasticity of two particles $i$ and $j . d_{i j}^{*}$ represents the equivalent diameter between the two particles $i$ and $j$, equal to $\frac{d_{j} d_{i}}{d_{j}+d_{i}}$. Since the radius of the nanoparticles is assumed to be equal, we defined the diameter between two particles to be equal to $\mathrm{R}$.

\section{Drag Force}

At a small scale the viscous forces are dominant, and the Reynolds number is very small $(\operatorname{Re} \ll 1)$. The drag model is represented as:

$$
F_{\text {drag }_{i}}=-6 \pi \mu R v_{p_{i}}
$$

where $R$ is equal to the radius of the nanoparticles, which is the same for all particles. Therefore, the drag force affecting each particle is only a function of the velocity of each particle.

\section{SimULATION PlATFORM FOR CAPTURING MNPS}

The MATLAB software has been used to develop the computational platform. The particles are randomly scattered in a fluid environment. The properties of nanoparticles and the environment are given in Table 1.

Table 1

(a) Fluid Properties (b) Nanoparticles Properties

\begin{tabular}{|c|c|c|}
\hline \multicolumn{3}{|c|}{ (a) Fluid Properties } \\
\hline Parameter & Value & Unit \\
\hline Density & 1050 & $\mathrm{Kg} / \mathrm{m}^{3}$ \\
\hline Viscosity & 0.004 & $\mathrm{~Pa} . \mathrm{s}$ \\
\hline $\begin{array}{c}\text { Permeability } \\
\text { Coefficient }\end{array}$ & 1 & - \\
\hline Temperature & 293.15 & $K$ \\
\hline
\end{tabular}

\begin{tabular}{|c|c|c|}
\hline \multicolumn{3}{|c|}{ (b) } \\
\hline Parameter & Value & Unit \\
\hline Radius1 & 500 & $\mathrm{~nm}$ \\
\hline Radius2 & 200 & $\mu \mathrm{m}$ \\
\hline Density & 5180 & $\mathrm{Kg} / \mathrm{m}^{3}$ \\
\hline Permeability & $1.257 \times 10^{-6}$ & $\mathrm{~V} \cdot \mathrm{s} /(\mathrm{A} \cdot \mathrm{m})$ \\
\hline
\end{tabular}

\section{A. Experimental Setup}

The experimental setup for the rotating field is shown in Fig.2. The region of interest is $10 \mathrm{~mm}$ (diameter) at the center of the actuation system. Two neodymium magnets (N42, 20 $\mathrm{mm}$ diameter by $10 \mathrm{~mm}$ width) are positioned by using a holder in a constant distance. The distance of the magnet holder can be varied to generate diverse magnetic fields. In this study, the magnet holder had a constant distance $(60 \mathrm{~mm})$ to generate a $25.46 \mathrm{mT}$ magnetic field. The maximum (9.7\%) and average (4.4\%) variation in the magnetic field was measured. An Arduino microcontroller, with a Motor Drive Controller (L298N), was used to control the field frequency, and the Iron particles with a radius $\leq 200 \mu \mathrm{m}$ were used in the experiments. 

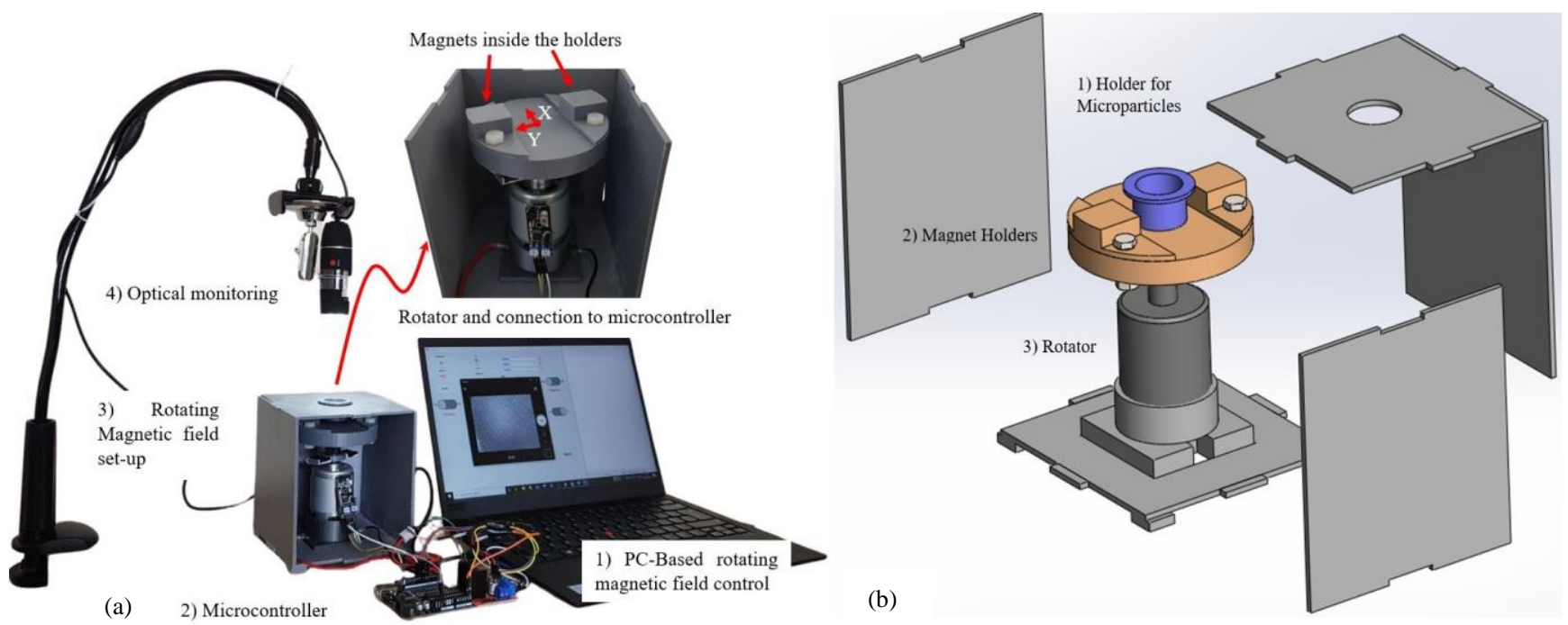

Figure 2. (a) Experimental setup for the swarm of the nanoparticles under a rotating magnetic field, the region of interest is considered as a circle with $10 \mathrm{~mm}$ diameter, (b) The schematic of the experimental setup

This experimental setup is not fully homogeneous. It has more inhomogeneity compares to Helmholtz coils (with $<1 \%$ or $<5 \%$ homogeneity). However, the effect of the magnetic field on the particles is assumed to be homogeneous due to the negligible impact of inhomogeneity in this setup.

Aggregated particles move in a chain form inside the region of interest. The aggregates are oriented along the direction of the magnetic field and rotated in response to the external field. For the rotating field of 5 to $10 \mathrm{~Hz}$, the aggregates rotate with the field without a change in their geometrical shape (remained as chain shape), with an increase in the field frequency to 20 $\mathrm{Hz}$, the chain shaped aggregates change to the sphere shaped formation. Finally, in higher frequencies (30 and $40 \mathrm{~Hz}$ ), dispersed sphere shaped aggregates are observed. The experiments have been carried out for $\mathrm{n}=3$ samples, and the results were compared with the simulation results.

\section{B. Comparison between simulation platform and experimental results}

In this study, two different systems are used to compare the simulation results. The results of [25] are used for smaller particles with a radius in the range of $100-500 \mathrm{~nm}$. The experimental setup (Fig. 2) is used to compare the simulation results of bigger particles $(<=200 \mu \mathrm{m})$. Although the platform can simulate magnetic force, the field is assumed to be homogeneous with a zero gradient, which results in the magnetic force to be zero $\left(F_{m_{i}}=0\right)$. The effects of dipoles on the particles play the key role in the motion of particles.

A criterion should be defined to study the particle concentration and the swarm behavior of the MNPs. The particle dispersion and aggregation are considered as the criterion to analyze different modes in swarm of the nanoparticle concentration.

For dispersion and aggregation, the criterion is particle dispersion change (PDC) and is defined as:

$$
d_{t}=\frac{\sum_{i}^{N} \sqrt{\left(x_{i}-\bar{X}_{c}\right)^{2}+\left(y_{i}-\bar{Y}_{c}\right)^{2}}}{L N}
$$

where, $\bar{X}_{c}$ and $\bar{Y}_{c}$ are considered the coordinates of the center of the region of interest. $x_{i}$ and $y_{i}$ show the coordinates of the particle $\mathrm{i}, d_{t}$ represents the dispersing at time $\mathrm{t}$, and $\mathrm{L}$ is the length and width of the range.

$$
\bar{d}_{\left(t_{1}, t_{2}\right)}=\frac{d_{t_{1}}-d_{t_{2}}}{d_{t_{1}}} \times 100
$$

where, $d_{t_{1}}$ and $d_{t_{2}}$ indicate dispersing at time $t_{1}$ and $t_{2} \cdot \bar{d}$ shows the PDC in the time span between $t_{1}$ and $t_{2}$.

$$
\begin{aligned}
& f_{x}=\sin \omega(t) \\
& f_{y}=\cos \omega(t)
\end{aligned}
$$

where $f_{x}$ specifies the field size ratio in the x-direction and $f_{y}$ is the field size ratio in the y-direction. $\omega$ gives us the frequency, which is equal to $\omega_{0}+\alpha t$. $\alpha$ and $\omega_{0}$ are two constant values.

Four different swarm modes have been observed for the accumulation of nanoparticles experimentally [25]. To analyze the swarm behavior and compare the experimental and numerical results, a parameter called the ideal percentage of dispersion changes (IPDC) has been introduced. This parameter is similar to the PDC, but it is calculated by the difference between the particle dispersion and ideal dispersion at time $\mathrm{t}$ (Eq. 11). Ideal dispersion is the minimum dispersion that particles can have. By using image processing, the number of particles in each image can be estimated. Using the number of obtained particles, the ideal dispersion in each image is calculated, which is approximately 0.7 in all 4 cases. Then, using image processing and computational algorithm, the particle dispersion in each image is calculated and shown in the first column of the Table 2 . 

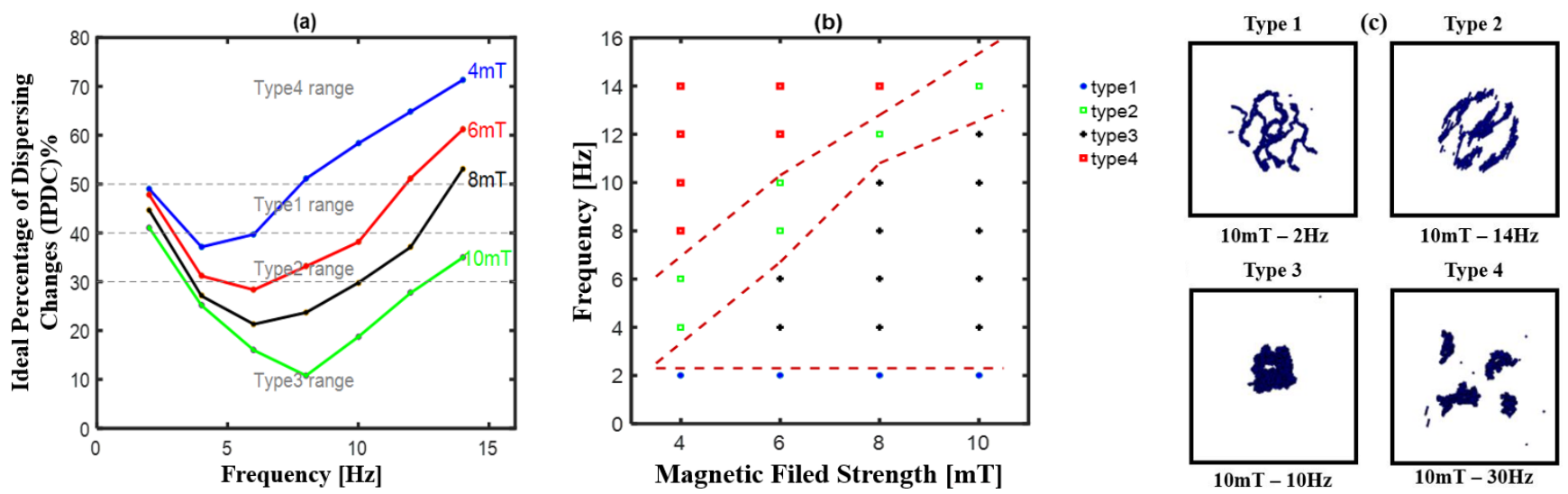

Figure 3. (a) The relationship between the IPDC with the frequency and the magnetic field strength is shown. The range of each swarm mode is shown by the fold line, (b) The phase diagram shows four different swarm behaviors in field and frequency changes. Swarm behaviors are categorized into four main types and are displayed in different colors. (c) Swarm behavior in four different states after $1 \mathrm{~s}$ of applying a magnetic field.
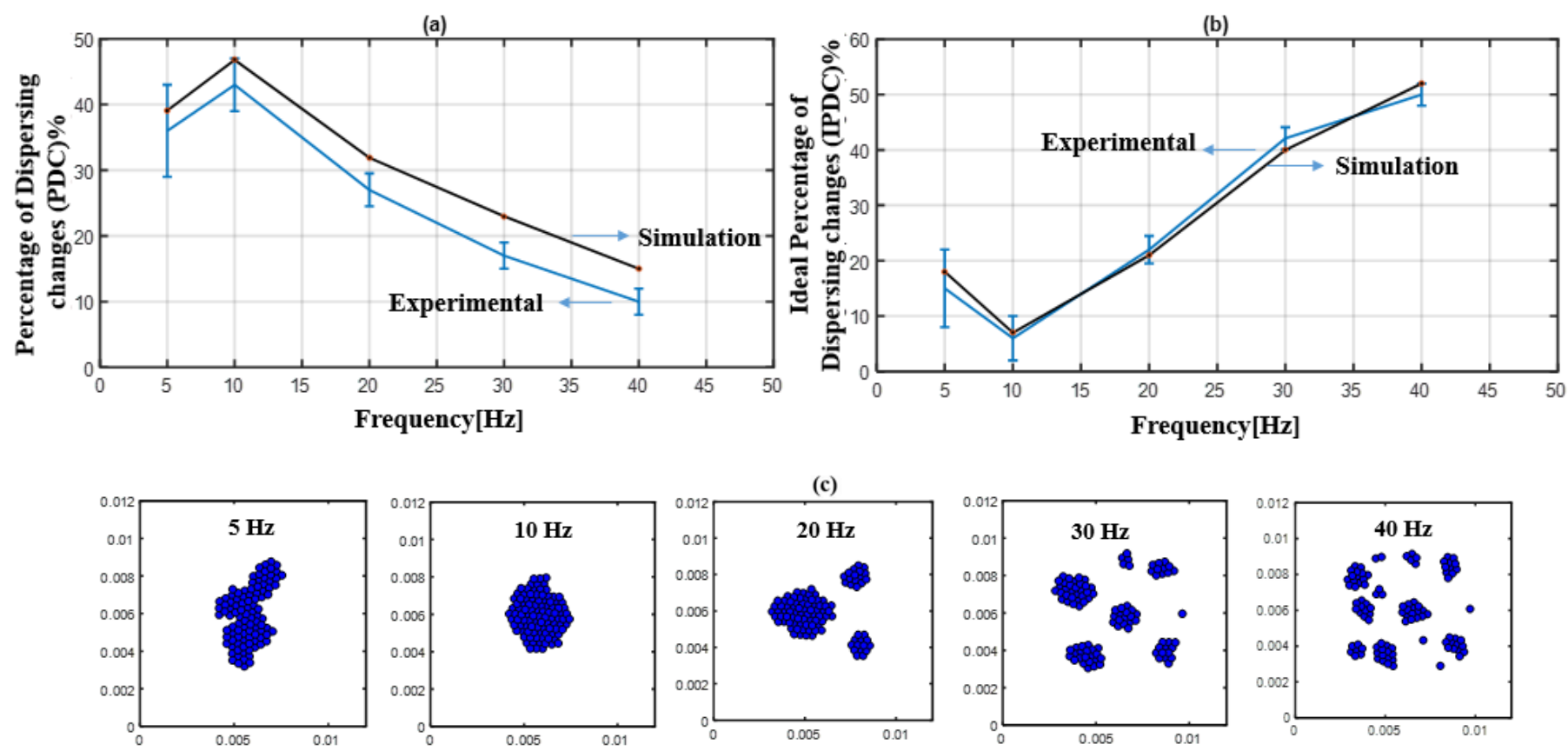

Figure 4. (a)The PDC values per frequency in the simulation and experimental results. (b)The IPDC values per frequency in the simulation and experimental results. (c) Swarm behavior in five different frequencies after $2.5 \mathrm{~s}$ of applying a magnetic field.

Table 2

The dispersion values and IPDCs associated with the experimental result are given, and according to the experimental values, the range of IPDCs for different types of swarm behavior is determined.

\begin{tabular}{|c|c|c|c|}
\hline Type & Dispersion & IPDC & Allowed Range \\
\hline 1 & 1.25 & $44 \%$ & $40 \%-50 \%$ \\
\hline 2 & 1.09 & $35 \%$ & $30 \%-40 \%$ \\
\hline 3 & 0.97 & $27 \%$ & $\leq 30 \%$ \\
\hline 4 & 1.53 & $54 \%$ & $\geq 50 \%$ \\
\hline
\end{tabular}

The simulation for 28 different modes of field effect on 1000 particles with $d_{t=0}=0.53$ and $d_{\text {Ideal }}=0.17$ is shown in Fig. 3. The results of the swarm behavior type in these 28 simulations (Fig. 3 (b)) has an average consistency of $\% 75$ with the experimental results in [25].
To compare the simulation and the experimental results, 100 particles with a radius of $200 \mu \mathrm{m}$ are used. By image processing, the dispersion of the initial and the final states is calculated. The comparison between PDC at different frequencies for the experimental and simulation results are shown in Fig. 4 (a). In this diagram, both curves for the numerical and experimental results show a similar behavior. However, at any frequency, the PDC values in the experimental results are less than the numerical results. The difference between the numerical and experimental results in the five considered frequencies varies between 5 to $15 \%$. The variation in the results can be linked to the initial dispersion. In the simulations, the initial dispersion of the particles is assumed to be random which is different from the initial dispersion of the particles in the experiments. Since PDC is obtained from the percentage of the change between the initial and final dispersion of particles, even with a similar final state, the PDC values will 
be different if the initial dispersion is different. The IPDC parameter is also used to study the results (Fig. 4-b). IPDC is independent of the initial particle dispersion. In the Fig. 4-b, the curve of the experimental and numerical results has a similar behavior, and the variation between the results is less than 5\% which indicates a good agreement between the experiment and simulation.

\section{STUDY OF EFFECTIVE PARAMETERS}

In this section, we study the effective parameters in the swarm nanoparticle capturing and different swarm modes. These parameters have been chosen based on the available experimental setups (e.g. Helmholtz system [25], MiniMag [31], OctoMag [32], [33]). In all studies, we use the PDC parameter to compare the effect of various parameters.

If the initial dispersion of the nanoparticles is insufficient (the distance between the particles is small), the effect of the field on the particles will not be significant because the particles stick together immediately. In this condition, the amount of dispersing in the initial and final state will be low (5-a). As a result of the lower PDC, the aggregated swarm type 3 is generated (Fig. 3). On the other hand, if the dispersion of the nanoparticles in the fluid is high (the distance between the nanoparticles will be high), the rotating field cannot create the aggregated MNPs. The dipole force is inversely proportional to

(a)

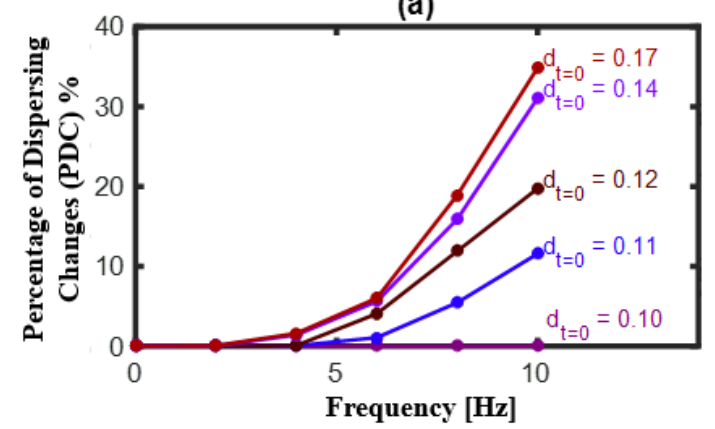

the fourth power of the particle distance $\left(\mathrm{F}_{\mathrm{dip}} \propto \frac{1}{\hat{\mathrm{r}}^{4}}\right)$, so the increase in the particle proximity reduces the dipole force and affects the aggregate (5-b).

The effect of MNPs radius change is shown in the Fig. 6. If the initial dispersion is sufficient (introduced in the Fig. 5), the dispersion approaches to the ideal dispersion (Fig. 6 (a)). Also, in all three cases, after a rotation of the field $(0.1 \mathrm{~s})$, the dispersion value remains constant.

The PDC of the different radius in various magnetic field strength (MFS) is similar. The small difference can be explained by increasing the mass and the dipole force (Fig. 6 (b)). If the initial dispersion is the same, the PDC will be different for the different radius (Fig. 6 (c)).

All simulations are done with the assumption that the particles have an equal radius. In Fig 6 (c) (fold line), the radius of the particles in the simulation is ranging between 300 500 $\mathrm{nm}$ (the average radius of the particles is $398 \mathrm{~nm}$ ). The result of this condition is close to the results of the particles with $400 \mathrm{~nm}$ radius. In Figure 6-c, similar to the Figure 6-a, after $0.1 \mathrm{~s}$, the dispersion remains constant.

In Fig. 7, It is shown that increasing the magnetic field strength will cause more particles to concentrate. Thus, it reduces the dispersion of particle, and increases the PDC of the particles.

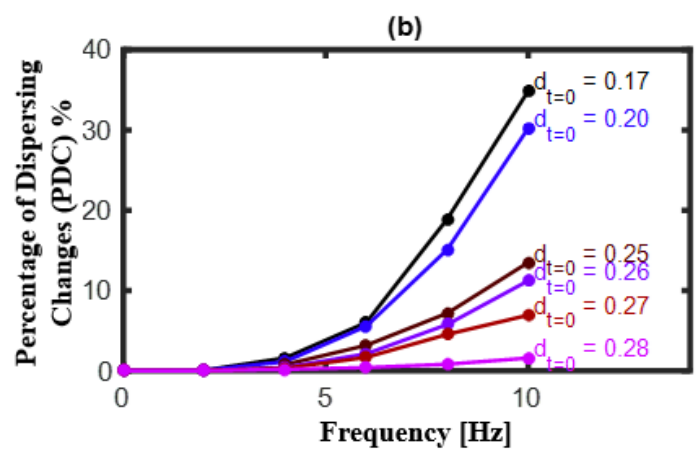

Figure 5: Magnetic field frequency effects on the PDC for 10 different conditions of the different initial dispersion is shown. (a) Ideal dispersion for 250 particles with a radius of $500 \mathrm{~nm}, \mathrm{PDC}$ is directly linked to the increase of dispersion from initial to 0.17. (b) further increase in the initial dispersion over 0.17 reduces the $\mathrm{PDC}$, and $d_{t=0}>0.28, \bar{d} \approx 0$.

(a)

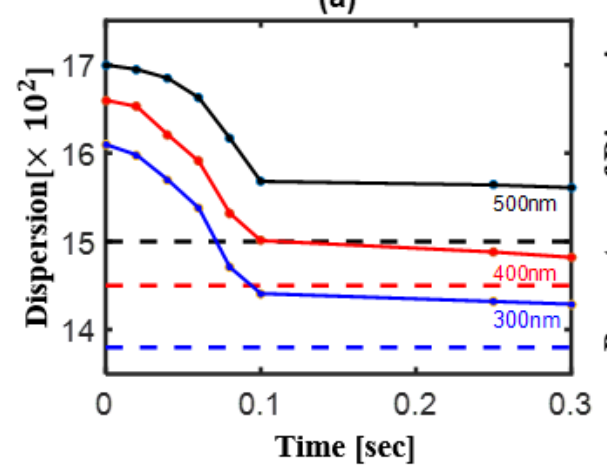

(b)

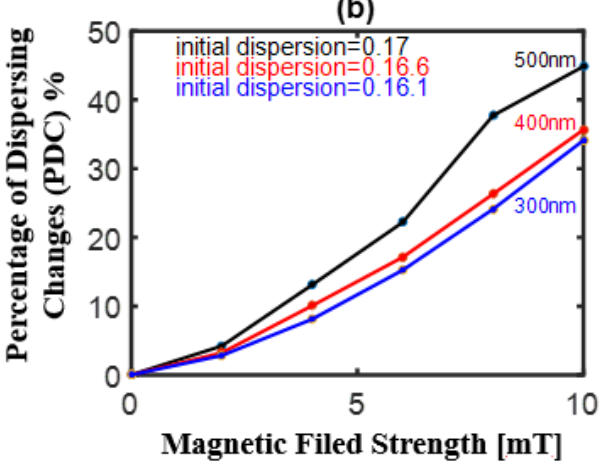

(c)

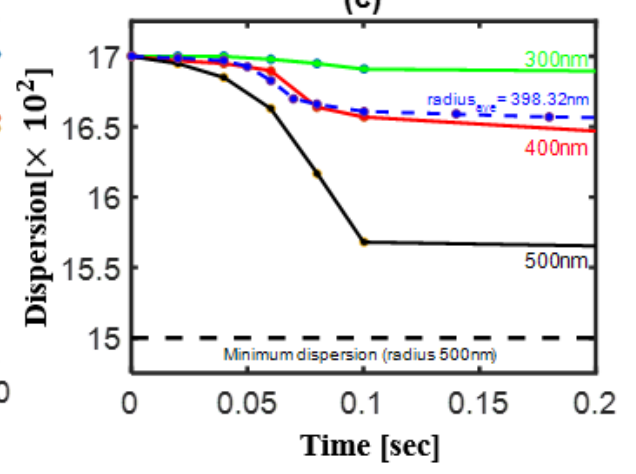

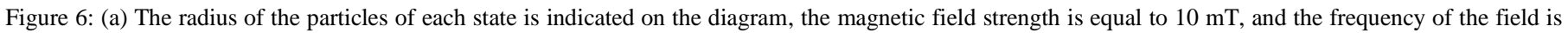

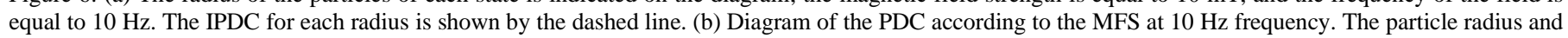

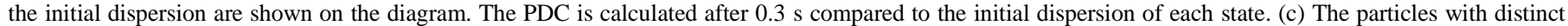
diameters in the range of 300 to $500 \mathrm{~nm}$ have been used, and the average particle radius is $398.3 \mathrm{~nm}$ (fold line). 
Initially, the increase in the frequency resulted in the final dispersion increase. However, after reaching the maximum dispersion, the particles do not find enough time to have a full rotation, which leads to a decrease in the final dispersion, and the PDC declines.

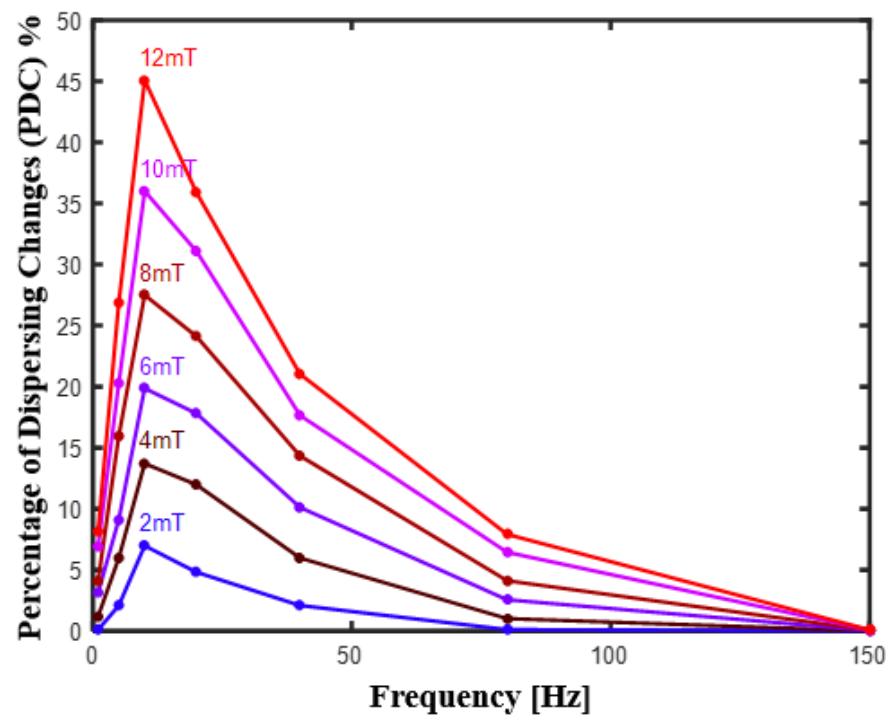

Figure 7: Diagram shows the effect of the frequency and intensity of the magnetic field on the PDC of the nanoparticles. All the cases are for the 250 particles with the same initial dispersion equal to 0.17 , and the PDC is calculated after $1 \mathrm{~s}$

\section{CONCLUSION}

The simulation platform can demonstrate swarm behavior numerically. The results obtained from the simulations are in good agreement with the experimental results. To analyze the behavior of the MNPs under the magnetic field, effective static parameters such as initial dispersion, MFS, frequency, and nanoparticle diameter were investigated.

Considering the effect of the different parameters on the type of the particle swarm behavior, the initial particle dispersion and excitation time can be considered as the most efficient parameters in the type of the particle swarm behavior. If the initial dispersion is below $(<0.12)$ or above $(>0.25)$ a threshold, the aggregate swarm is not generated.

The simulations showed that although the role of the magnetic field strength is higher than frequency, in the lower frequencies, high dispersion cannot be achieved. The effect of the magnetic field intensity on the PDC was studied, using 100 , 200, 500, 1000, and 2000 particles, which in all cases, the greater the magnitude of the field, led to less dispersion. Overall, the simulation platform can predict the parameters for a successful swarm mode generation to be used in the experimental studies.

The main objective of this work was modeling the particle swarm in different conditions. In the future work, we will study the separation and the effective parameters in the separation control. Considering the clinical importance of MNPS $<100 \mathrm{~nm}$ in hyperthermia and drug delivery, unmodeled forces which can be effective on particles $<100 \mathrm{~nm}$, are another interesting area of the research. Introducing these forces in the modeling can lead to a more generalized platform.

\section{REFERENCES}

[1] I. R. Edwards and J. K. Aronson, "Adverse drug reactions: Definitions, diagnosis, and management," Lancet, vol. 356, no. 9237, pp. 1255-1259, 2000, doi: 10.1016/S0140-6736(00)02799-9.

[2] A. S. Lübbe, C. Alexiou, and C. Bergemann, "Clinical applications of magnetic drug targeting," J. Surg. Res., vol. 95, no. 2, pp. 200206, 2001, doi: 10.1006/jsre.2000.6030.

[3] E. Taylor and T. J. Webster, "Reducing infections through nanotechnology and nanoparticles.," Int. J. Nanomedicine, vol. 6, pp. 1463-1473, 2011, doi: 10.2217/nnm.11.123.

[4] H. Kempe and M. Kempe, "The use of magnetite nanoparticles for implant-assisted magnetic drug targeting in thrombolytic therapy," Biomaterials, vol. 31, no. 36, pp. 9499-9510, 2010, doi: 10.1016/j.biomaterials.2010.07.107.

[5] Y. Ju et al., "Monodisperse Au-Fe2C Janus Nanoparticles: An Attractive Multifunctional Material for Triple-Modal ImagingGuided Tumor Photothermal Therapy," ACS Nano, vol. 11, no. 9, pp. 9239-9248, 2017, doi: 10.1021/acsnano.7b04461.

[6] Z. Zhou, Z. Shen, and X. Chen, "Tale of Two Magnets: An Advanced Magnetic Targeting System," ACS Nano, vol. 14, no. 1, pp. 7-11, 2020, doi: 10.1021/acsnano.9b06842.

[7] D. De Lanauze, O. Felfoul, J. P. Turcot, M. Mohammadi, and S. Martel, "Three-dimensional remote aggregation and steering of magnetotactic bacteria microrobots for drug delivery applications," Int. J. Rob. Res., vol. 33, no. 3, pp. 359-374, 2014, doi: $10.1177 / 0278364913500543$.

[8] S. Earnshaw, "On the Nature of the Molecular Forces which Regulate the Constitution of the Luminiferous Ether," Trans. Cambridge Philos. Soc., vol. 7, p. 97, Jan. 1848.

[9] B. Ramaswamy et al., "Movement of magnetic nanoparticles in brain tissue: Mechanisms and impact on normal neuronal function," Nanomedicine Nanotechnology, Biol. Med., vol. 11, no. 7, pp. 18211829, 2015, doi: 10.1016/j.nano.2015.06.003.

[10] M. Park, T. A. Le, A. Eizad, and J. Yoon, "A Novel Shared Guidance Scheme for Intelligent Haptic Interaction Based Swarm Control of Magnetic Nanoparticles in Blood Vessels," IEEE Access, vol. 8, pp. 106714-106725, 2020, doi: 10.1109/ACCESS.2020.3000329.

[11] H. Liu et al., "Magnetic resonance monitoring of focused ultrasound / magnetic nanoparticle targeting delivery of therapeutic agents to the brain," vol. 107, no. 34, pp. 15205-15210, 2010, doi: 10.1073/pnas.1003388107.

[12] M. Vonthron, V. Lalande, G. Bringout, C. Tremblay, and S. Martel, "A MRI-based integrated platform for the navigation of microdevices and microrobots," IEEE Int. Conf. Intell. Robot. Syst., pp. 1285-1290, 2011, doi: 10.1109/IROS.2011.6048274.

[13] M. Latulippe and S. Martel, "Dipole field navigation: Theory and proof of concept," IEEE Trans. Robot., vol. 31, no. 6, pp. 1353-1363, 2015, doi: 10.1109/TRO.2015.2489518.

[14] S. Martel, "Combining pulsed and DC gradients in a clinical MRIbased microrobotic platform to guide therapeutic magnetic agents in the vascular network," Int. J. Adv. Robot. Syst., vol. 10, 2013, doi: $10.5772 / 53513$.

[15] A. K. Hoshiar, T. A. Le, F. U. Amin, M. O. Kim, and J. Yoon, "A novel magnetic actuation scheme to disaggregate nanoparticles and enhance passage across the blood-brain barrier," Nanomaterials, vol. 8, no. 1, 2018, doi: 10.3390/nano8010003.

[16] F. U. Amin et al., "Osmotin-loaded magnetic nanoparticles with electromagnetic guidance for the treatment of Alzheimer's disease," Nanoscale, vol. 9, no. 30, pp. 10619-10632, 2017, doi: 10.1039/c7nr00772h.

[17] T. A. Le, X. Zhang, A. K. Hoshiar, M. O. Kim, and J. Yoon, "Simulation studies of a novel electromagnetic actuation scheme for focusing magnetic micro/nano-carriers into a deep target region," AIP Adv., vol. 7, no. 5, 2017, doi: 10.1063/1.4977018.

[18] Y. Liu et al., "a Novel Approach to Accumulate Superparamagnetic Particles in Aqueous Environment using Time-Varying Magnetic."

[19] A. K. Hoshiar, T. A. Le, and J. Yoon, "Electromagnetic actuation scheme for swarm of magnetic nanoparticles steering in multibifurcation," Proc. MARSS 2019 4th Int. Conf. Manip. Autom. Robot. Small Scales, vol. 61005, pp. 1-6, 2019, doi: 10.1109/MARSS.2019.8860939.

[20] A. K. Hoshiar, T. A. Le, P. Valdastri, and J. Yoon, "Swarm of magnetic nanoparticles steering in multi-bifurcation vessels under fluid flow," J. Micro-Bio Robot., 2020, doi: 10.1007/s12213-020- 
00127-2.

[21] Y. Gao, A. Van Reenen, M. A. Hulsen, A. M. De Jong, M. W. J. Prins, and J. M. J. Den Toonder, "Disaggregation of microparticle clusters by induced magnetic dipole-dipole repulsion near a surface," Lab Chip, vol. 13, no. 7, pp. 1394-1401, 2013, doi: 10.1039/c3lc41229f.

[22] J. Yu and L. Zhang, "Characterising of mobile vortex-like paramagnetic nanoparticle swarm: From a single vortex to multiple vortices," 2017 IEEE 17th Int. Conf. Nanotechnology, NANO 2017, pp. 293-296, 2017, doi: 10.1109/NANO.2017.8117390.

[23] J. Yu, T. Xu, Z. Lu, C. I. Vong, and L. Zhang, "On-Demand Disassembly of Paramagnetic Nanoparticle Chains for Microrobotic Cargo Delivery," IEEE Trans. Robot., vol. 33, no. 5, pp. 1213-1225, 2017, doi: 10.1109/TRO.2017.2693999.

[24] J. Yu, D. Jin, K. F. Chan, Q. Wang, K. Yuan, and L. Zhang, “Active generation and magnetic actuation of microrobotic swarms in biofluids," Nat. Commun., vol. 10, no. 1, pp. 1-12, 2019, doi: 10.1038/s41467-019-13576-6.

[25] J. Yu, L. Yang, and L. Zhang, "Pattern generation and motion control of a vortex-like paramagnetic nanoparticle swarm," Int. J. Rob. Res., vol. 37, no. 8, pp. 912-930, 2018, doi: 10.1177/0278364918784366.

[26] H. Xie et al., "Reconfigurable magnetic microrobot swarm: Multimode transformation, locomotion, and manipulation," Sci. Robot., vol. 4, no. 28, pp. 1-15, 2019, doi: 10.1126/scirobotics.aav8006.

[27] P. Vartholomeos and C. Mavroidis, "In Silico Studies of Magnetic Microparticle Aggregations in Fluid Environments for MRI-Guided Drug Delivery," IEEE Trans. Biomed. Eng., vol. 59, no. 11, pp. 3028-3038, Nov. 2012, doi: 10.1109/TBME.2012.2213340.

[28] A. K. Hoshiar, T. A. Le, F. U. Amin, M. O. Kim, and J. Yoon, "Studies of aggregated nanoparticles steering during magneticguided drug delivery in the blood vessels," J. Magn. Magn. Mater., vol. 427, no. November, pp. 181-187, 2017, doi: 10.1016/j.jmmm.2016.11.016

[29] P. I. Dolez, Nanoengineering: Global Approaches to Health and Safety Issues. 2015.

[30] J. J. Abbott, E. Diller, and A. J. Petruska, "Magnetic Methods in Robotics," Annu. Rev. Control. Robot. Auton. Syst., vol. 3, no. 1, pp. 57-90, 2020, doi: 10.1146/annurev-control-081219-082713.

[31] S. Jeon et al., "Improving guidewire-mediated steerability of a magnetically actuated flexible microrobot," Micro Nano Syst. Lett., vol. 6, no. 1, 2018, doi: 10.1186/s40486-018-0077-y.

[32] S. Lee et al., "Fabrication and Characterization of a Magnetic Drilling Actuator for Navigation in a Three-dimensional Phantom Vascular Network," Sci. Rep., vol. 8, no. 1, pp. 1-9, 2018, doi: 10.1038/s41598-018-22110-5.

[33] A. K. Hoshiar, S. Jeon, K. Kim, S. Lee, J. Y. Kim, and H. Choi, "Steering algorithm for a flexible microrobot to enhance guidewire control in a coronary angioplasty application," Micromachines, vol. 9, no. 12, pp. 1-13, 2018, doi: 10.3390/mi9120617. 